\title{
Orientation of the Liquid Crystals of Polyribonucleotide Complexes in a Static Magnetic Field
}

\author{
Eisaku IrzuKA \\ Institute of High Polymer Research, Faculty of Textile Science and Technology, \\ Shinshu University, Ueda 386, Japan.
}

(Received October 17, 1977)

\begin{abstract}
KEY WORDS Liquid Crystal / Polyribonucleotide Complex / Magnetic-Field Orientation / Magnetic Susceptibility /
\end{abstract}

We reported that polymer helices of certain polyribonucleotide complexes in concentrated solutions form liquid crystals of the nematic type, in which double-stranded helices of poly(A) $\cdot \operatorname{poly}(\mathrm{U})$ and triple-stranded helices of poly(A) 2 poly(U) are converted, on standing, to the cholesteric type. ${ }^{1}$ The pitch of the cholesteric liquid crystals of these two complexes changes in a static magnetic field and this change varies with the angle which the layers of molecular alignment make with respect to the magnetic field, indicating that the polymer helices are magnetically susceptible. This susceptibility appears to be higher in the direction perpendicular to the helical axes than in the direction parallel to the helical axes. ${ }^{1,2}$ I should like to report in this communication that the nematic liquid crystals of poly(A) $\operatorname{poly}(\mathrm{U})$ and poly(A). 2 poly(U) can be oriented in a static magnetic field.

X-Ray diffraction photographs of the polymer films prepared from the nematic solutions of $\operatorname{poly}(\mathrm{A}) \cdot \operatorname{poly}(\mathrm{U})$ and $\operatorname{poly}(\mathrm{A}) \cdot 2 \operatorname{poly}(\mathrm{U})$ in a static magnetic field of 25 kilogausses are shown in Figure 1. The photographs taken at the N-position have two equatorial reflections corresponding to $3.3 \AA$ and $3.8 \AA$ spacings, respectively and two meridian reflections corresponding to $4.6 \AA$ and $5.8 \AA$ spacings, respectively in both complexes (Figure $1 \mathrm{~N}$ ). The $3.3-\AA$ spacing is considered to be related to the stacked bases at right angles to the axes of helical polymers in the B-configuration, ${ }^{3}$ which is a helical form, found in deoxyribonucleic acid, with the phosphate groups outside and a translation of $3.4 \AA$, and a screw rotation of $36^{\circ}$ between the hydrogen-bonded base pairs. Among the complexes of synthetic polyribonucleotides, this configuration was first found in poly(A). poly(U) by Rich and his coworkers. ${ }^{4}$ (A small difference in the spacing corresponding to the base stacking is not significant. The $3.3-\AA$ reflection was observed in poly(C) - poly(I) helices by the same authors. ${ }^{5}$ ) The $\mathrm{X}$-ray result suggests that the polymer helices are oriented in a direction perpendicular to the magnetic field since the specimen films were placed so that the magnetic field was vertical when taking the photographs; furthermore, their magnetic susceptibilities are stronger in the direction perpendicular than parallel to the helical axes. This agrees with the idea obtained from the magnetic-field effect on the cholesteric state concerning the direction of maximum susceptibility. $^{2}$

The photographs taken at the C-position are similar to those taken at the N-position, except that the inner reflections are well separated at the C-position, and that their patterns are tilted by $90^{\circ}$ compared with those at the N-position (Figure 1C). These indicate that the polymer helices are certainly oriented perpendicularly to the magnetic field, and also show the presence of double orientation in the polymer helices. A strong equatorial reflection corresponding to $12.4 \AA$ spacing in poly(A). $\operatorname{poly}(\mathrm{U})$ and $12.8 \AA$ spacing in poly(A). 2 poly(U), each average of four observations, is related to the polymer-chain interval. If polymer helices are aligned parallel to the direction of external forces, as in the stretched film ${ }^{6}$ and the electrically 
Poly (A) · poly (U)

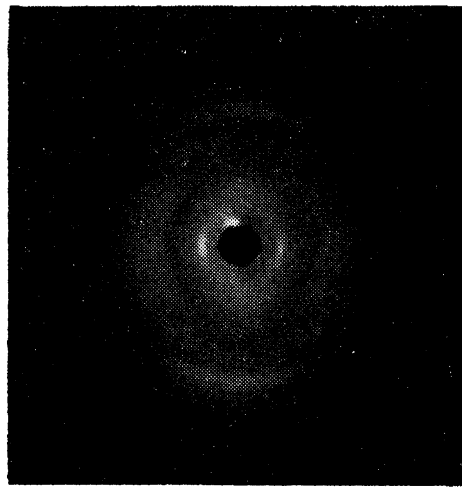

$(\mathrm{N})$

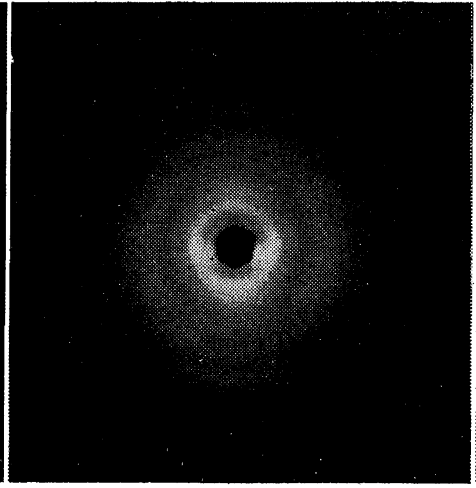

(E)

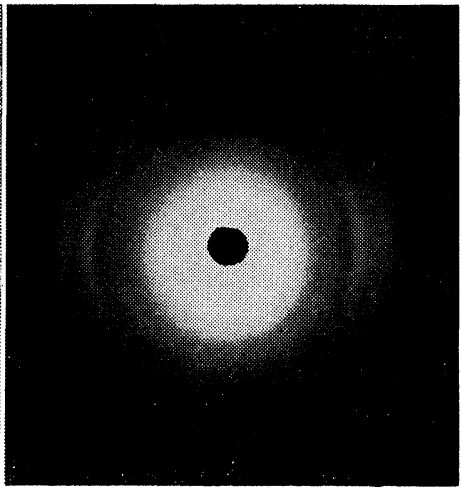

(C)

Poly (A) $\cdot 2$ poly (U)

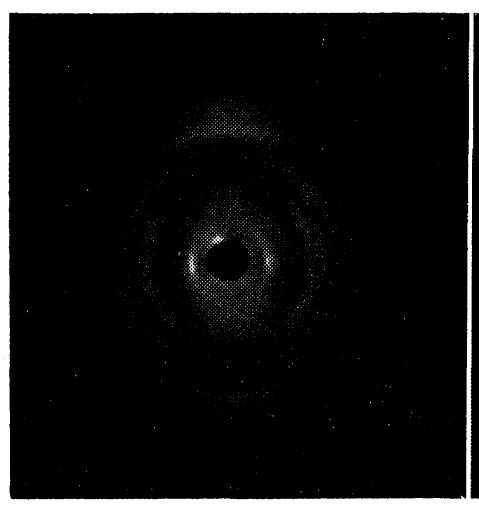

(N)

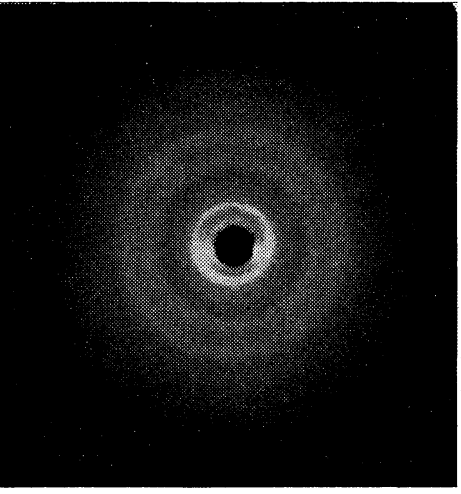

(E)

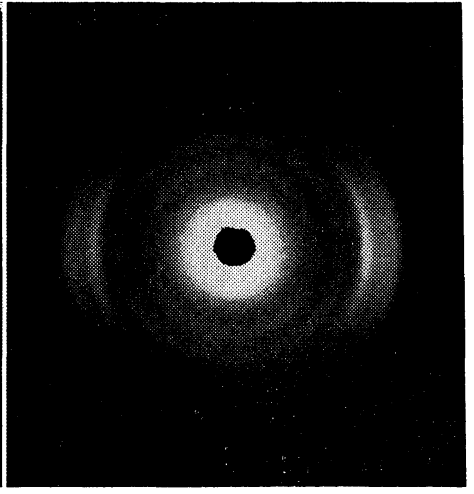

(C)

Figure 1. X-Ray diffraction photographs of the magnetically oriented films of polyribonucleotide complexes: field strength, 25 kilogausses; relative humidity, 50-\% RH; (N) beam normal to polymer film, direction of magnetic field vertical; (E) beam parallel to surface of polymer film, direction of magnetic field vertical; (C) beam along direction of magnetic field, polymer film in a vertical plane.

oriented (liquid crystalline) solid film ${ }^{7}$ of $\alpha$ poly $(\gamma-$ benzyl-L-glutamate), these patterns are obtained at the E-position and not at the C-position.

These polypeptide specimens are well doubleoriented and show the X-ray diffraction patterns typical of the hexagonal arrangement of polymer helices at the C-position. The photographs taken at the E-position corresponding to those of $\alpha$ poly( $\gamma$-benzyl-L-glutamate) taken at the C-position are certainly different from those taken at the $\mathrm{N}$ - and C-positions; however, their patterns are, even though an indication of the hexagonal array of polymer helices is seen at the $12-13 \AA$ reflection, almost of the Debye-Scherrer ring type, suggesting that the double orientation of polymer helices are not noticeable (Figure 1E). The 3.3- $\AA$ reflection that should not appear in perfect orientation is still seen; however, it is naturally very weak especially in poly(A) 2 poly(U).

In a magnetic field of 10 kilogausses or more, the nematic solutions of $\operatorname{poly}(\mathrm{A}) \cdot \operatorname{poly}(\mathrm{U})$ and poly $(\mathrm{A}) \cdot 2$ poly(U) showed larger refractive indices when the electric vector of incident light was parallel to the magnetic field than when it was perpendicular to the magnetic field. Since the polyribonucleotide helices show negative birefringence, ${ }^{8}$ this agrees with the conclusion derived from the X-ray results concerning the direction of 
orientation of the polymer helices.

Strangely enough, the nematic solutions first formed after preparation did not show the magnetic-field orientation. Only the nematic solutions, reversed from the cholesteric solutions by shearing stresses, could be oriented. Poly(A) - poly(U) recovered the cholesteric texture more rapidly than $\operatorname{poly}(\mathrm{A}) \cdot 2 \operatorname{poly}(\mathrm{U})$ which completely recovered the cholesteric texture one day after shearing, and so was this polymer complex even during the solution was dried in the magnetic field. This is because the cholesteric texture becomes more stable against a magnetic field in a higher polymer concentration. $^{2}$ Thus, thicker films of poly(A) $\cdot \operatorname{poly}(\mathrm{U})$ requiring more time for preparation were not oriented. Other polyribonucleotide complexes such as $\operatorname{poly}(\mathrm{G}) \cdot \operatorname{poly}(\mathrm{C})$, $\operatorname{poly}(\mathrm{C}) \cdot \operatorname{poly}(\mathrm{I})$ and poly(A) 2 poly(I) could not be magnetically oriented, which except poly(C)-poly(I) were highly viscous and could not flow. The lack of fluidity may be one of the reasons for this, as in the case of the cell-free liquid crystals of deoxygenated sickle-cell hemoglobin. ${ }^{9} \quad \operatorname{Poly}(\mathrm{C}) \cdot \operatorname{poly}(\mathrm{I})$ contains some spherulitic structures in the nematic texture, ${ }^{1}$ which may be another reason for this.

Blyumenfel'd and his coworkers were the first to study the magnetism of nucleic acids ${ }^{10}$ and concluded that this magnetism was peculiar to these compounds and not due to any impurities present. ${ }^{11}$ This idea was supported by some and was denied by others, and is still not settled. ${ }^{12}$ I believe from these results that the magnetic anisotropy shown by polyribonucleotide complexes is evidence of the presence of genuine magnetism in these polymers. Shearing stresses may help produce independent rod-like molecular clusters and the magnetic-field orientation can occur because these molecular culsters have large, induced (magnetic) dipole moments as in the liquid crystals of polypeptides. $^{13-15}$

\section{EXPERIMENTAL}

Concentrated solutions of poly(A) $\cdot \operatorname{poly}(\mathrm{U})$ and poly(A) 2 poly $(\mathrm{U})$ having polymer concentration of $5.5 \mathrm{vol} \%$ were kept at room temperature for several weeks to insure full growth of the cholesteric liquid crystals. Each solution was sheared to break down the cholesteric texture (and to resume the nematic texture), and then to put in a cubic cell $1 \times 1 \mathrm{~cm}$ inside and placed between the poles of an electromagnet, being a modified model of JM151 manufactured by Japan Electron Optics Laboratory Co., Ltd. The solution was then dried in a static magnetic field $(25 \mathrm{kG})$ parallel to the surface of the solution and at room temperature $(50-\% \mathrm{RH})$ to obtain a polymer film of thickness a few micrometers. In doing so, the solution was allowed to start drying about $2 \mathrm{hr}$ after application of the magnetic field and to be dried up for several hours by gentle air-flashing with an electric fan. The preparation of polyribonucleotide complexes have been described elsewhere. ${ }^{1,2}$ X-Ray diffraction photographs of the polymer films thus obtained were taken with a Rigaku Denki Geigerflex $\left(35 \mathrm{kV}, 25 \mathrm{~mA}, \mathrm{Cu}-\mathrm{K}_{\alpha}\right.$ line) from three directions (see the caption to Figure 1). For taking measurements at the $\mathrm{E}$ - and C-positions, the polymer films were cut into strips which were pasted with gum arabic face to face to attain sufficient width and suitable thickness.

\section{REFERENCES}

1. E. Iizuka and J. T. Yang, Proc. Symp. Ordered Fluids and Liquid Crystals, 174th National Meeting of the American Chemical Society, Chicago, Ill., Aug. 1977, to be published by Plenum Press.

2. E. Iizuka, submitted to Polym. J.

3. J. D. Watson and F. H. C. Crick, Nature, 171, 737 (1953).

4. A. Rich and D. R. Davies, J. Am. Chem. Soc., 78, 3548 (1956).

5. D. R. Davies and A. Rich, ibid., 80, 1003 (1958).

6. C. H. Bamford, W. E. Hanby and F. Happey, Proc. Roy. Soc., A205, 39 (1951).

7. E. Iizuka, Biochim. Biophys. Acta, 175, 457 (1969).

8. G. B. B. M. Sutherland and M. Tsuboi, Proc. Roy. Soc., A239, 446 (1957).

9. E. Iizuka, Proceedings of 6th International Liquid Crystals Conference, Part D, Molecular Crystals and Liquid Crystals, in press.

10. L. A. Blyumenfel'd, A. E. Kalmanson, and Sheng Péi-Ken, Dokl. Akad. Nauk. SSSR, 124, 1144 (1959).

11. L. A. Blyumenfel'd and V. A. Benerskii, ibid., 133, 1451 (1960).

12. H. Morimoto, Buturi (in Japanese), 17, 587 (1962).

13. E. Iizuka, Biochim. Biophys. Acta, 243, 1 (1971).

14. E. Iizuka, Mol. Cryst. Liq. Cryst., 27, 161 (1974).

15. E. Iizuka, $A d v$. Polym. Sci., 20, 79 (1976). 